\title{
A Preliminary Comparison of Three Tourniquet Instructions for Just-in- Time Guidance of a Simulated Tourniquet Application
}

Bethany Lowndes, Katherine Forsyth, Erik Prytz, Carl-Oscar Jonson, Amro Abdelrahman, Sztajnkrycer Matthew, Walter Franz, III, Renaldo Blocker and Susan Hallbeck

\section{Book Chapter}

Pre-Print

N.B.: When citing this work, cite the original article.

Part of: Proceedings of the Human Factors and Ergonomics Society 2017 Annual Meeting, 2017, pp. 1076-1080.

DOI: https://doi.org/10.1177/1541931213601873

Copyright: Authors and Human Factors and Ergonomics Society, HFES

Available at: Linköping University Institutional Repository (DiVA)

http://urn.kb.se/resolve?urn=urn:nbn:se:liu:diva-141997 


\title{
A Preliminary Comparison of Three Tourniquet Instructions for Just-in-Time Guidance of a Simulated Tourniquet Application
}

\author{
Bethany R. Lowndes, $\mathrm{PhD}^{1,2}$, Katherine L. Forsyth, $\mathrm{PhD}^{1,2}$ Erik Prytz, $\mathrm{PhD}^{3,4}$, Carl-Oscar Johnson, $\mathrm{PhD}^{4}$, \\ Amro Abdelrahman MBBS ${ }^{1,2}$, Matthew D. Sztajnkrycer, MD, $\mathrm{PhD}^{6}$, Walter B. Franz, III,MD ${ }^{5}$, Renaldo \\ C. Blocker, $\mathrm{PhD}^{1,2} \mathrm{M}$. Susan Hallbeck, $\mathrm{PhD}^{1,2}$

\begin{abstract}
${ }^{1}$ Mayo Clinic, Robert D. and Patricia E. Kerns Center for the Science of Health Care Delivery, Rochester, MN; ${ }^{2}$ Mayo Clinic, Linköping, Sweden; ${ }^{4}$ Linköping University, Center for Disaster Medicine and Traumatology, Department of Clinical and Experimental Medicine, Linköping, Sweden; ${ }^{5}$ Mayo Clinic, Department of Medicine, Rochester, MN; ${ }^{6}$ Mayo Clinic, Department of Family Medicine, Rochester, MN
\end{abstract} \\ Department of Health Sciences Research, Rochester, MN; ${ }^{3}$ Linköping University, Department of Computer and Information Science,
}

The American College of Surgeons (ACS) and the Department of Homeland Security (DHS) encourage the use of tourniquets as part of the "Stop the Bleed" campaign. Both have provided instructions for layperson tourniquet application in order to save the life of a hemorrhaging trauma victim. This study sought to compare the impact of using ACS and DHS instructions with manufacturer-provided instructions for the completion of simulated tourniquet application steps. Thirty surgical trainees completed a simulated tourniquet application with one of the three instruction sets. Their completion time and success for each step were measured. Participants that received ACS instructions failed the fewest number of steps $(\mathrm{p}<$ 0.01 ) and completed the task faster (Mean (SD) $=70$ (33) seconds) compared to those with the manufacturer-provided instructions $(\mathrm{p}<0.01)$. Tourniquet instruction sets need to be refined in order to optimize the success rate of just-in-time guidance for tourniquet application.

\section{Tourniquet Use}

\section{INTRODUCTION}

Massive hemorrhage control is a critical first step in saving the life of a trauma victim. When a first responder arrives on scene, there are many steps to ensure safety of the victim and responder. When direct pressure either cannot stop bleeding or is not feasible due to the situation, a tourniquet may be necessary. Until recently, there has been limited support from medical practitioners in using tourniquets to stop hemorrhage, due to concern for tissue ischemia and compromise (Lee, Porter, \& Hodgetts, 2007). Based upon the military experience with tourniquet applications, and faced with an increased potential for mass casualty events in the United States, prehospital care standards are changing (American College of Surgeons, 2016; Frangou, 2016; U.S. Department of Homeland Security, 2015). Military experience demonstrates that tourniquet use saves lives; even after many hours of tourniquet use, tissues that were deprived of blood can still heal without the need for amputation (Beekley et al., 2008; Kragh Jr, Baer, \& Walters, 2007; Kragh Jr et al., 2009; Lakstein et al., 2003). Although the civilian literature is sparse, similar results have been noted (Scerbo et al., 2016; Stiles, Cook, \& Sztajnkrycer, 2017).

The American College of Surgeons (ACS) has initiated the "Stop the Bleed" campaign in conjunction with the Department of Homeland Security (DHS) in order to promote and educate the public on tourniquet use in trauma situations (Frangou, 2016). There is a vision-at present time with minimal implementation - to provide access to tourniquets in public places just like the deployment of Automated External Defibrillators (AEDs) (Jacobs, Burns, Langer, \& Kiewiet de Jonge, 2016). However, very little is known about how to provide just-in-time emergent guidance for tourniquet application.
AEDs have been optimized to provide clear pragmatic guidance to anyone using one in an emergency situation. AED instruction has also been included in lifesaving courses such as those offered by the American Red Cross. These pre-emptive trainings can lead to increased confidence and success (Mancini et al., 2009; Yeung, Okamoto, Soar, \& Perkins, 2011). However, instructions provided with the AED packaging may be adequate to instruct new untrained users in the just-in-time application (Yeung, et al., 2011), even during an emergency.

Ideally, tourniquet instructions would be optimized to guide any individual through successful application including first-time users. This would increase the chance of tourniquet success by a layperson in an emergency situation. If tourniquets are placed in public areas along with AEDs starting immediately, there will be limited options for just-intime instruction. Between ACS, DHS and the directions provided with the commercially available tourniquet used in this study (Combat Application Tourniquet Version 7[CAT7]), several potential instructions are available for lay providers for tourniquet use. To our knowledge, these instructions have not been compared or evaluated for their effectiveness in providing just-in-time training for successful tourniquet use. The goal of this study was to determine if medical trainees could complete 10 identified steps in tourniquet application based on the instructional guidance of one of three (DHS, ACS, CAT-7) instruction sets currently available.

\section{METHODS}

In this prospective trial, general surgery residents (postgraduate year 1 or PGY-1), medical students, and research trainees (postgraduate researchers with a medical degree) participated in an educational event to train and test their knowledge and surgical skills while rotating through 
several skill stations. This study focused on tourniquet use in a trauma simulation and compared three different instruction sets for impact on application of a commercially available tourniquet.

\section{Instrumentation}

The commercially available CAT-7 $(\mathrm{C} \cdot \mathrm{A} \cdot \mathrm{T}$ Resources, LLC; Rock Hill, SC) was used for this simulation. This tourniquet consisted of: (A) a Velcro constricting band for placement on a limb, (B) a windlass for further tightening the tourniquet, (C) a clip to secure the windlass, and (D) a Velcro strap to ensure the windlass remained secure and allowed for a time notation (Figure 1). A manikin lower limb with a simulated open fracture and resultant uncontrolled hemorrhage provided a consistent model for a patient that would require a tourniquet (Figure 2).

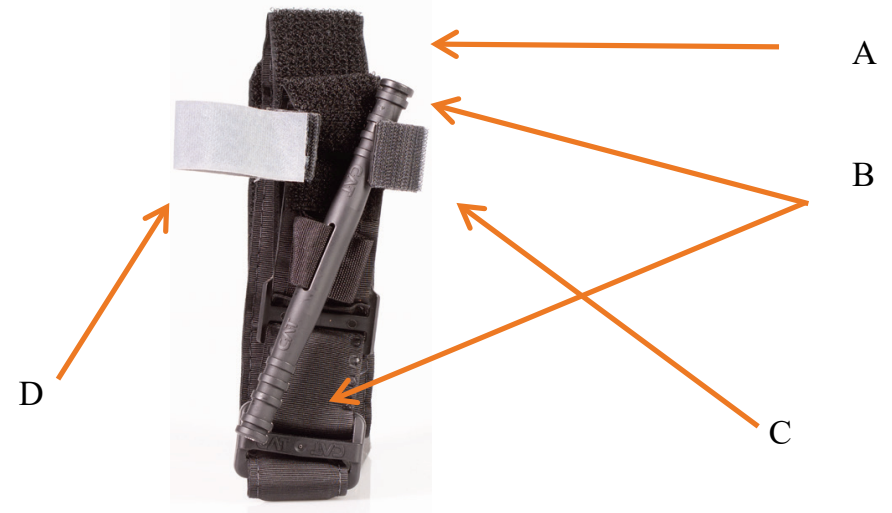

Figure 1. CAT-7 Tourniquet

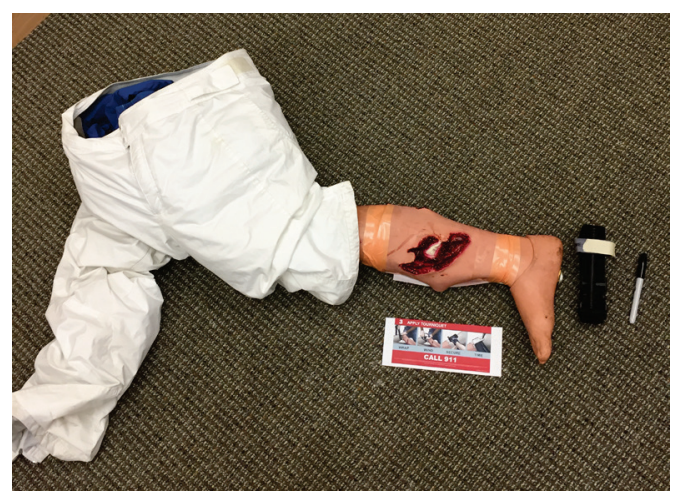

Figure 2. Simulated lower limb fracture with uncontrolled bleeding showing tourniquet and instruction placement.

\section{Participant recruitment and setting}

A convenience sample of surgical PGY-1 residents, medical students, and surgical research trainees was collected during a bi-annual surgical skills assessment event (Farley, Greenlee, Haigh, Cook, \& Enger, 2009). The 30 participants rotated through different surgical skills stations. This study includes the results from one of eighteen 7-minute station during this surgical skills event. Participants were randomized to the three different instruction sets according to gender and level of education/role (resident, medical student, research trainee) for stratification.

\section{Instruction sets}

Three different instruction sets developed by ACS, DHS, and the manufacturer of the CAT-7 were utilized for this study (Figure 3A-C). Only steps specific for tourniquet use and placement were included in the simulation event (e.g., steps such as direct pressure were excluded) to focus solely on tourniquet use and instruction utility.
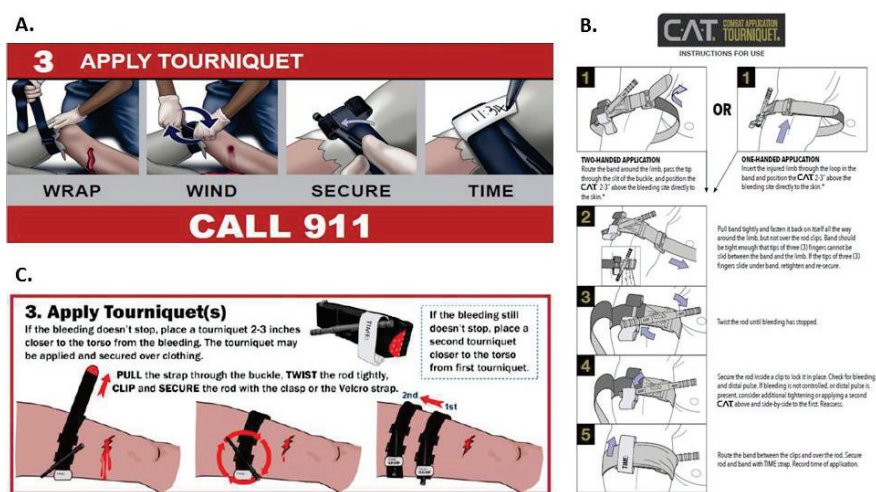

Figure 3. Tourniquet instructions provided by: A. ACS, B. CAT-7, C. DHS.

\section{Tourniquet Application Steps}

Using the Combat Medic Advanced Skills Training (CMAST: Student Reference, 2005) along with the manufacturer-provided instructions on how to use the FDAapproved CAT-7 tourniquet $(\mathrm{C} \bullet \mathrm{A} \bullet \mathrm{T}$ Resources, LLC; Rock Hill, SC), we were able to identify 10 steps for tourniquet application (Table 2). The only critical steps the trainees were asked to perform to increase the chance of survival (as described by the CMAST) were to place the tourniquet properly (proximal to the wound) and stop the bleed. To the best of our knowledge there is no standardized military or civilian sequence of steps for tourniquet application specifically for the CAT-7. We followed CMAST (Combat Medic Advanced Skills Training (CMAST): Student Reference, 2005) closely but included a few steps that were only mentioned in the CAT-7 manufacturer instructions. Using the steps identified from these resources, we sought to evaluate the ability of the participants to meet these steps based on the provided instruction set.

\section{Data collection}

Participants first completed a baseline questionnaire about previous experience and confidence with tourniquets. When entering the room for the task, the simulation set up (Figure 2) was behind a screen so the participants remained blinded to the task. Upon entry, they were briefed on the incident, provided with verbal instructions, and asked about any questions they had prior to starting their task (See Table 1). Participants were not informed of the study purpose, which was to evaluate the impact of the instruction sets on performance. After completing the briefing, they were permitted to go past the screen and begin the task. Immediately upon passing the screen, they saw the simulation set-up as seen in Figure 2 and their time to "stop the bleed" began. They were randomized to one of the three instruction sets and allotted 3 minutes to complete the tourniquet 
application to qualify as successful application but were asked to verbally indicate task completion. The study was approved by the Mayo Clinic Institutional Review Board.

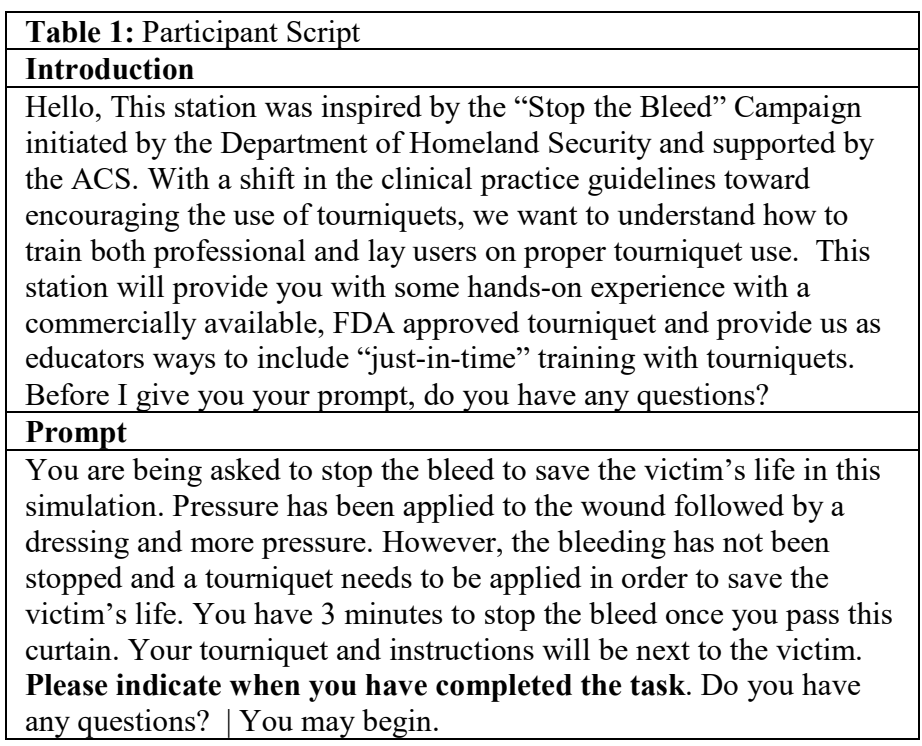

\section{Evaluation}

Two human factors engineers conducted the set-up, introduction, and evaluation. The baseline questionnaire focused on the participants' experience with a tourniquet. Participants were not randomized by their previous training or experience since the researchers collected this information at the time of the study. During the tourniquet application, one human factors engineer observed for completion of 3 steps (Table 2, steps 7-9) and the second human factors engineer recorded the time to completion. After the simulated tourniquet application by the first trainee and while the next participant was applying a tourniquet, the second human factors engineer evaluated the success of the task by the first trainee. All steps were deemed pass or fail based on the ability of the participant to complete the steps with the provided instruction set.

\begin{tabular}{|l|l|l|}
\hline \multicolumn{3}{|l|}{ Table 2. Explanation of Identified Tourniquet Steps } \\
\hline $\mathbf{1}$ & Subjective & Tightness of the Windless \\
\hline $\mathbf{2}$ & Subjective & Less than 3 fingers fit under secured strap \\
\hline $\mathbf{3}$ & Objective & Placement: $\geq 2$ inches above wound \\
\hline $\mathbf{4}$ & Objective & Windless secured \\
\hline $\mathbf{5}$ & Objective & Extra Strap secured in Clip \\
\hline $\mathbf{6}$ & Objective & Time strap secured over Clip \\
\hline $\mathbf{7}$ & Observation & Windless/Rod Twisted \\
\hline $\mathbf{8}$ & Observation & Did not cross the wound when applying \\
\hline $\mathbf{9}$ & Observation & Re-thread the Tourniquet \\
\hline $\mathbf{1 0}$ & Objective & Notation of Time or Tourniquet in use \\
\hline
\end{tabular}

\section{Data analysis}

Descriptive statistics were performed to compare participant groups and failure rates based on instruction type provided. Performance Time and Percentage of Failed Steps were compared across instruction type groups using ANOVAs at an alpha $=0.05$ level with Tukey post-hoc analyses.

\section{RESULTS}

The 30 participants consisted of 17 male and 13 female participants, 1 medical student, 26 residents, and 2 research trainees. Thirteen $(43.3 \%)$ of the participants had prior training in tourniquet use, predominantly through the ACS-Advanced Trauma Life Support (ATLS) course. Average prior experience included performing two tourniquet applications to a maximum of 5 applications. There was no specification of simulated vs clinical application. Participants with previous experience self-rated initial higher confidence and familiarity with tourniquet application in the baseline questionnaire.

\section{Failed Steps}

In an ANOVA for the independent variable of Instruction type (ACS, DHS, CAT-7) by level of training (Trained, Untrained), instruction type was significant, $\mathrm{F}(2,25)$ $=6.182, \mathrm{p}<0.01$, but no effect of training was shown, $\mathrm{F}(1$, $25)=7.128, p=.314$, nor effect due to interaction between training and instruction type, $\mathrm{F}(2,25)=1.762, \mathrm{p}=0.192$ on the number of failed steps. Further Tukey post-hoc tests showed that the participants following the ACS instructions had a significantly lower percentage of failed steps as compared to the participants who received the CAT-7 manufacturer instructions, $\mathrm{p}<0.01$ as well as compared to the participants who received the DHS instructions, $p=0.024$, see Figure 4.

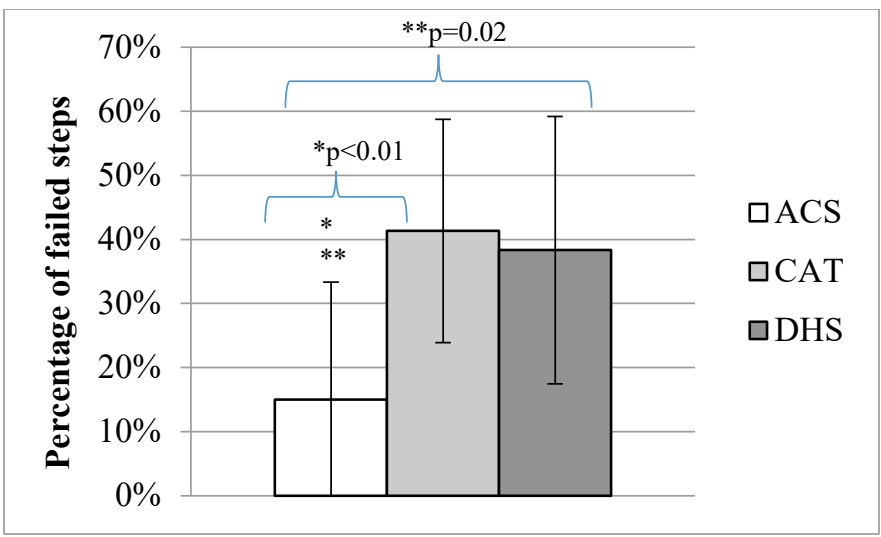

Figure 4. Average percentage of failed steps from the Identified Tourniquet Steps based on instruction type (ACS, CAT-7, or DHS).

Although there were no statistically significant differences in the percentage of participants who failed for each step, $55-70 \%$ (variation based on the instruction set received) of the participants failed step 2 and did not tighten the strap tight enough that three fingers could not slide under it once fastened with Velcro, potentially not stopping hemorrhage. In addition, $60-90 \%$ of the participants failed Step 9 of Table 2 and did not re-thread the tourniquet.

\section{Speed of Application}

Overall, participants completed the task in mean (SD) 101 (40) seconds with a maximum of time of 180 seconds 
$(\mathrm{n}=5,17 \%)$ at which time they were stopped (these participants had finished the task but had not yet verbalized completion) and minimum of 35 seconds $(n=1)$. When stratified by instruction type, the fastest average completion time was with the ACS instruction set at 70 (33) seconds. The results from the ANOVA comparing performance (dependent variable, completion time in seconds) by the type of instruction (ACS, DHS, CAT-7) and by the level of tourniquet training (Trained, Untrained) showed a significant effect of instructions, $\mathrm{F}(2,25)=7.128, \mathrm{p}<0.01$, but no effect of training, $F(1,25)=7.128, p=0.314$. To follow up with this ANOVA, the Tukey post-hoc tests showed that the ACSgroup had a significantly shorter average application time as compared to the CAT-7 group ( $\mathrm{p}<0.01$, Figure 5).

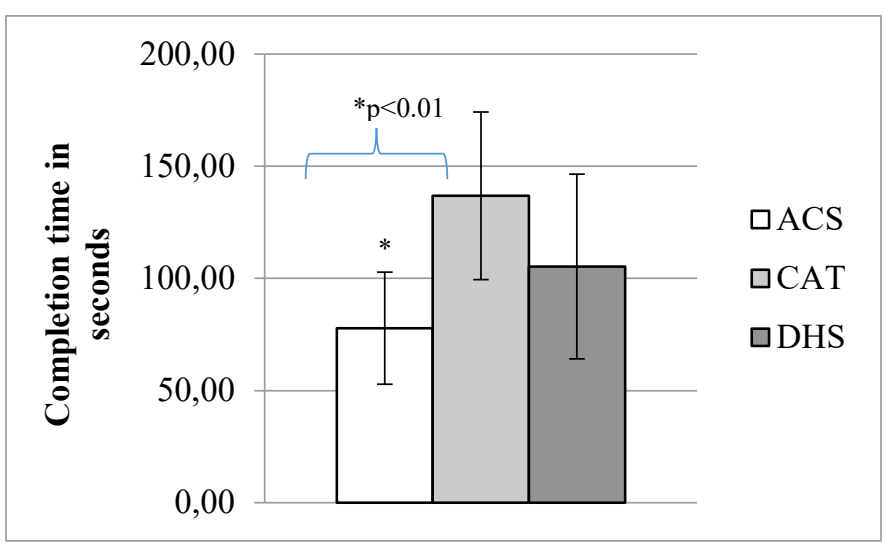

Figure 5. Comparison of task completion time (sec) based on instruction type received.

\section{DISCUSSION}

This study compared participant performance of tourniquet application with three different instruction sets. While tourniquet use is currently being promoted to the public (Frangou, 2016), this study questions the ability for medical students, surgical residents, and research trainees to follow currently published tourniquet application instructions. This is particularly concerning given the level of pre-existing medical knowledge available to these participants which would likely be absent in the general public. Participant performance (i.e., successfully completed steps) and speed of the simulated tourniquet application varied based on the instruction set received. Based on this pilot study, the ACS instructionswhich are more pictorial (Figure 3.B) - yielded the best application performance and speed results. However, there are still many improvements that could be made to the instruction set in order to improve clarity and ideally performance of the tourniquet application task.

Despite the fact that $43 \%$ of participants had prior tourniquet training and therefore increased familiarity and confidence, the instruction sets did not impact the number of failed steps during the tourniquet application simulation. As shown in Figure 3, the ACS instruction set was very simple, had a straightforward flow, and was primarily graphic with very few words. This format most effectively conveyed instructions to the participants allowing them to successfully apply the tourniquet more rapidly than the CAT-7, which had considerable text in small font.
Comparing the instruction sets with participant ability to perform each step of Table 2, certain procedural steps were not well represented across any of the instruction sets. For example, on Step 2 (Table 2) where the strap should be tightened so that less than three fingers could slide under the strap once fastened with Velcro, participants performed poorly on this step regardless of the instruction set received. While wrapping the tourniquet strap around the limb is obvious for the ACS instructions (Figure 3A), there is no indication that three fingers should not fit under the strap as indicated in the CAT-7 instructions (Figure 3B). In the middle of a relatively large block of text in the CAT-7 instructions, the Step 2 metric is listed. However, many participants still failed this step when the received the CAT-7 instruction set. It is likely that they missed this step due to its location in the instruction set and the density of the surrounding text (Wogalter, Conzola, \& Smith-Jackson, 2002). It must be noted that the CAT-7 manufacturer instructions were not developed as a just-in-time training tool, but rather as a comprehensive reference to be used during formal hands-on training programs. However, without any other available instructions or previous training on tourniquet use, it may be used as a justin-time training tool for a layperson in an emergency situation. Finally, in the DHS instruction set (Figure 3C), there is simply a step indicating that the strap should be pulled through the buckle; there is no instruction or indication to use the Velcro to secure the strap nor an indication of how tight it should be secured. All three instruction sets failed to properly instruct over half of the participants, all of whom were medically trained.

The instruction for two-hand application is to rethread the strap proximal to the wound (Step 9). In the ACS instruction (Figure 3A), there is no instruction beyond simply wrapping the strap around the limb. In the CAT-7 instructions (Figure 3B) there are two different instructions with an "OR" between indicating one- or two-handed application. For the two-handed application portion there is a note in the text to" re-thread the strap". Finally, for the DHS instruction set (Figure 3C), there is nothing more than an instruction to pull the strap through the buckle. All three instruction sets failed to instruct a majority of the participants to re-thread the tourniquet.

For the speed of application, participants were provided three minutes to complete the tourniquet task. Some participants (17\%) used the full three minutes for the simulation. Although they had finished the application, they had not verbalized completion and were re-reading instructions or visually checking the tourniquet. In a real-life scenario, this should be within the time window needed to stop exsanguinating hemorrhage to save the life of the victim; however the time to exsanguination will be dependent on the type of bleed and the time between the initial trauma and the tourniquet application. Participants performed the task the fastest with the ACS instruction set. This was likely due to the fact that the instructions flowed well and had very few words to distract participants or require studying while completing the tourniquet application task. Participants' performance time with the DHS instruction set was not significantly slower than those with the ACS instruction set. This could be due to the 
fact that while the DHS instruction set had more words and a less straightforward flow, it too was a relatively graphic instruction set. The CAT-7 instruction set was the most cumbersome and time-consuming for the participants due to the excessive words and small pictures. This is true even though these were highly educated surgical trainees with good vision; something not necessarily representative of the general public accessing a tourniquet posted near and AED. However, it must be noted that the ACS and DHS instruction sets were specifically developed as just-in-time training tools, while the manufacturer instructions were not.

Overall, each instruction set did not adequately instruct users to complete some of the identified steps for justin-time tourniquet application. This pilot study demonstrated that without a standard tourniquet instruction set, it was difficult for even medically trained individuals to correctly and successfully apply a tourniquet. Given this finding, we speculate it will be equally or more difficult for laypeople or trained individuals who have not practiced this task recently to apply a tourniquet to stop hemorrhaging in a trauma victim, especially in an emergency. Refining instructions to reach performance efficiency analogous to the AED instructions will also aid in creating tourniquet education curricula ranging from trained medical professionals to the general public/laypeople.

As with every study, there were some limitations to this pilot study. First, the participants were not randomized to the three instruction sets based on prior tourniquet experience since they answered this question after randomization. This could have skewed some results based on the different instruction sets. However, the results of the ANOVA indicated no impact due to training. Second, since a manikin without a pulse or simulated bleeding was used, we could not confirm that the bleeding was stopped or enough pressure had been applied to stop the distal pulse. Therefore, despite failing some steps, participants could have successfully applied the tourniquet and ultimately completed the task of stopping the bleed. Additionally, this study did not require continual pressure on the wound during tourniquet application in order to more thoroughly evaluate usability. Finally, the steps were not tested for clinical validity or necessity. Therefore, some of the steps that we identified could be incorrect or unnecessary. Future studies that can measure a more accurate level of success will be able to better evaluate the instruction sets and the impact they have on the performance of tourniquet application and lifesaving.

\section{CONCLUSION}

With recent emphasis on hemorrhage control for victims with exsanguinating extremity trauma, a pragmatic instruction set is needed to guide both professional and untrained laypeople in the application of tourniquets. While the ACS instruction set provides some promising performance benefits, our study suggests that the instructions are fallible for just-in-time training. Simple instructions with detailed pictures instead of words will likely have more success in guiding individuals through successful tourniquet application. Simple modifications to the instructions could improve the success of placing tourniquets in publicly available locations immediately. These results could further be used to create an optimal instruction design for improved just-in-time tourniquet application success rates.

\section{ACKNOWLEDGEMENT}

This research was funded in part by the Mayo Clinic Robert D. and Patricia E. Kern Center for the Science of Health Care Delivery.

\section{REFERENCES}

American College of Surgeons. (2016). Did You Know? Stop the Bleed and BleedingControl.org. The Cutting Edge: Post-Clinical Congress 2016 Edition, from

https://www.facs.org/publications/newsletters/the-cuttingedge/postcc2016/dyk-stop-the-bleed

Beekley, A. C., Sebesta, J. A., Blackbourne, L. H., Herbert, G. S., Kauvar, D. S., Baer, D. G., .. . Group, s. C. S. H. R. (2008). Prehospital tourniquet use in Operation Iraqi Freedom: effect on hemorrhage control and outcomes. Journal of Trauma and Acute Care Surgery, 64(2), S28-S37.

Combat Medic Advanced Skills Training (CMAST): Student Reference. (2005) (Phase 2 ed.)

Farley, D., Greenlee, S., Haigh, L., Cook, J., \& Enger, T. (2009). Deliberate practice and feedback: the benefit of a Surgical Olympics. Journal of surgical education, 66(2), 63.

Frangou, C. (2016). 'Stop the Bleed' Campaign Focuses on Saving Lives With Simple Techniques New Course to Train Civilians as Immediate Responders During Mass Casualty Events; Surgeons Urged to Take Lead. General Surgery News.

Jacobs, L. M., Burns, K. J., Langer, G., \& Kiewiet de Jonge, C. (2016). The Hartford Consensus: A National Survey of the Public Regarding Bleeding Control. Journal of the American College of Surgeons, 222(5), 948-955. doi: http://dx.doi.org/10.1016/j.jamcollsurg.2016.02.013

Kragh Jr, J. F., Baer, D. G., \& Walters, T. J. (2007). Extended (16-hour) tourniquet application after combat wounds: a case report and review of the current literature. Journal of orthopaedic trauma, 21(4), 274-278.

Kragh Jr, J. F., Walters, T. J., Baer, D. G., Fox, C. J., Wade, C. E., Salinas, J., \& Holcomb, J. B. (2009). Survival with emergency tourniquet use to stop bleeding in major limb trauma. Annals of Surgery, 249(1), 1-7.

Lakstein, D., Blumenfeld, A., Sokolov, T., Lin, G., Bssorai, R., Lynn, M., \& Ben-Abraham, R. (2003). Tourniquets for hemorrhage control on the battlefield: a 4-year accumulated experience. Journal of Trauma and Acute Care Surgery, 54(5), S221-S225.

Lee, C., Porter, K. M., \& Hodgetts, T. J. (2007). Tourniquet use in the civilian prehospital setting. Emergency Medicine Journal, 24, 584-587. doi: $10.1136 / \mathrm{emj} .2007 .046359$

Mancini, M. E., Cazzell, M., Kardong-Edgren, S., Cason, C. L., Berryman, P., $\&$ Lukes, E. (2009). Improving workplace safety training using a self-directed CPR-AED learning program. Aaohn Journal, 57(4), 159-169.

Scerbo, M. H., Mumm, J. P., Gates, K., Love, J. D., Wade, C. E., Holcomb, J. B., \& Cotton, B. A. (2016). Safety and appropriateness of tourniquets in 105 civilians. Prehospital emergency care, 20(6), 712-722.

Stiles, C. M., Cook, C., \& Sztajnkrycer, M. D. (2017). A descriptive analysis of tactical casualty care interventions performed by law enforcement personnel in the state of Wisconsin, 2010-2015. Prehospital and disaster medicine, 1-5.

U.S. Department of Homeland Security. (2015). Presidential Policy Directive / PPD-8: National Preparedness Retrieved 02/09/2017, 2017, from https://www.dhs.gov/presidential-policy-directive-8-nationalpreparedness

Wogalter, M. S., Conzola, V. C., \& Smith-Jackson, T. L. (2002). Researchbased guidelines for warning design and evaluation. Applied ergonomics, 33(3), 219-230.

Yeung, J., Okamoto, D., Soar, J., \& Perkins, G. D. (2011). AED training and its impact on skill acquisition, retention and performance-a systematic review of alternative training methods. Resuscitation, $82(6), 657-664$. 